\title{
LIGHT CURVES COMPARISON: ROBOTIC OBSERVATORY VERSUS NON-ROBOTIC ONE
}

\author{
S. H. Hendi ${ }^{1}$ and F. Bahrani ${ }^{1}$
}

\begin{abstract}
Regarding the advanced robotic technology, one of the logical questions is the human roles in controlling observation devices which may affect the results of data. Here, we want to study the light curves of robotic telescopes and then compare it with a non-robotic one to find out the influence of technology/human on data accuracy.
\end{abstract}

\section{RESUMEN}

En cuanto a la tecnología robótica avanzada, uno de las preguntas lógicas son los roles humanos en el control de los dispositivos de observación que pueden afectar los resultados de los datos. Aquí nosotros estudiamos las curvas de luz de los telescopios robóticos y luego las comparamos con la obtenida por un telescopio no robótico para encontrar la influencia de la tecnología/acción humana en la precisión de los datos.

Key Words: light curves - methods: miscellaneous

\section{INTRODUCTION}

As it may be clear, instruments have an important effect on observation results. In this study, we want to see different of observations with robotic instruments and non-robotic ones. So, first, we will introduce our studied robotic observatory which is Waffelow Creek Observatory in Nacogdoches of Texas then we will introduce Biruni observatory that is a non-robotic observatory in Shiraz, Iran. After that, we will insert some information about our target which is an eclipsing binary star and compare the result of introduced robotic and non-robotic observatories by plotting both outcome light curves.

\section{WAFFELOW CREEK OBSERVATORY}

Waffelow Creek Observatory is a personal observatory in Nacogdoches, Texas (see Figures 1, 2 and 3). The idea of building this robotic observatory is belongs to its owner Edward J. Michaels from Stephen F. Austin State University. Due to the fact that he was so busy (teaching and directing a planetarium) and his university three telescopes were not automated, he has decided to build his own completely automated one three years ago. He wanted it to be robotic so it could efficiently collect CCD data for his research projects without a user to tend the telescope/camera all night. Most of the components used to assemble the observatory are readily available. Lots of assemblies required, especially building the roll-off roof structure.

\footnotetext{
${ }^{1}$ Physics Department and Biruni Observatory, College of Sciences, Shiraz University, Shiraz 71454, Iran (Hendi@shirazu.ac.ir, Bahrani-f@yahoo.com).
}

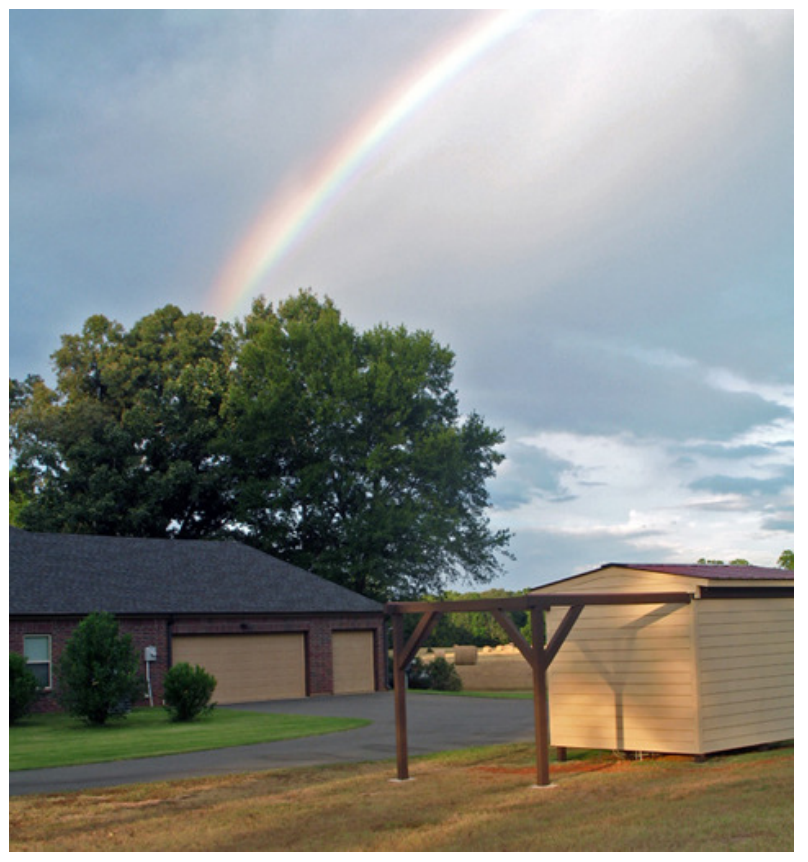

Fig. 1. A view of Waffelow Creek robotic Observatory in Nacogdoches, Texas.

\subsection{Major Components}

Here is a list of the major components with sources:

- Software Bisque Paramount MX robotic telescope mount and software. http://www. bisque.com/sc/pages/ParamountMX. aspx

- 14-inch RC TPO telescope 


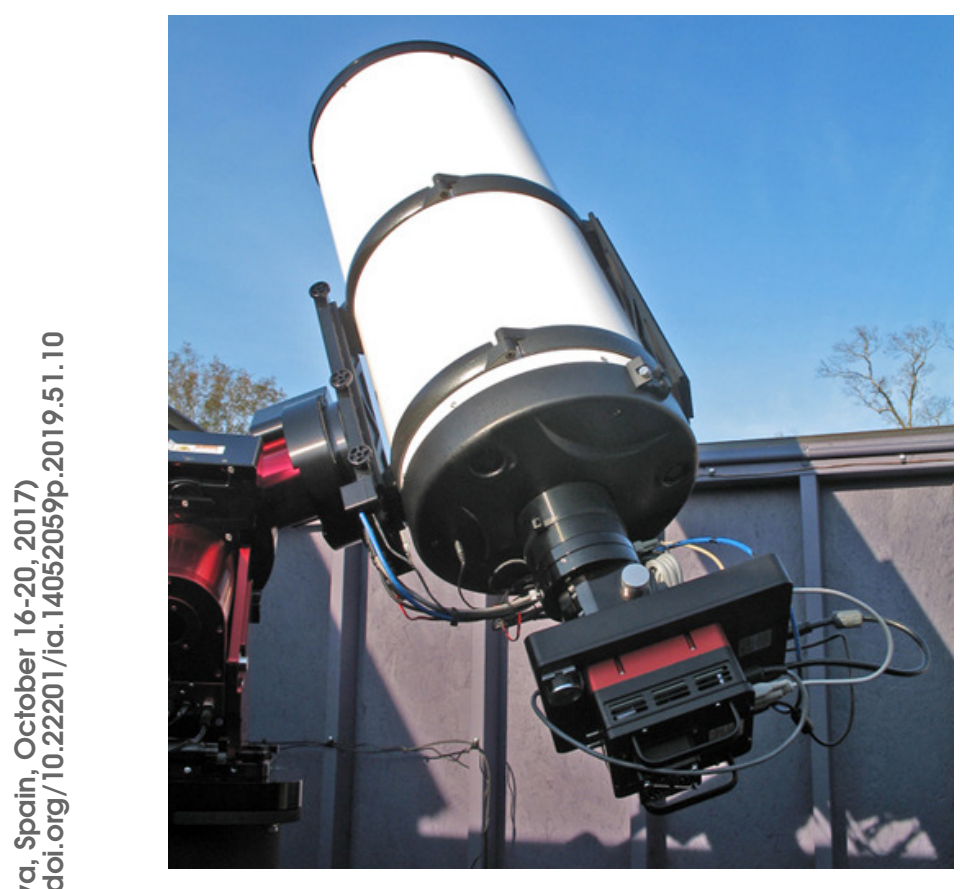

Fig. 2. Previous telescope of Waffelow Creek Observatory: $0.31 \mathrm{~m}$ telescope (which our data came from).

https://optcorp.com/collections/ ritchey-chretien-telescopes/products/ tpo-14-f8-rc-truss-tube-telescope

- OPTEC telescope focuser

https://optcorp.com/collections/

telescope-focusers/products/

optec-tcfs-3-3-id-temperature-compensatingfocuser

- SBIG STXL-6303E CCD camera with filter wheel and sets of Johnson BVRI and Sloan g', r' and I' photometric filters

http://diffractionlimited.com/product/ stxl-6303e/

http://www . astrodon.com/index.html

- Roof Control

http://jimstar11.com/mvocontrols.html

- Weather Sensors and Cloud Detection http://jimstar11.com/SkyAlert.html

- PC Computer with the following software packages:

"TheSkyX Professional Edition" for Telescope Control.

"SkyRoof" for roof control.

"SkyAlert" for weather alerts.

"MaxIm DL" for camera control.

"FocusMax" for automated focusing.

"ACP" for observatory control and acquiring images (http://acp.dc3.com/index2.html).

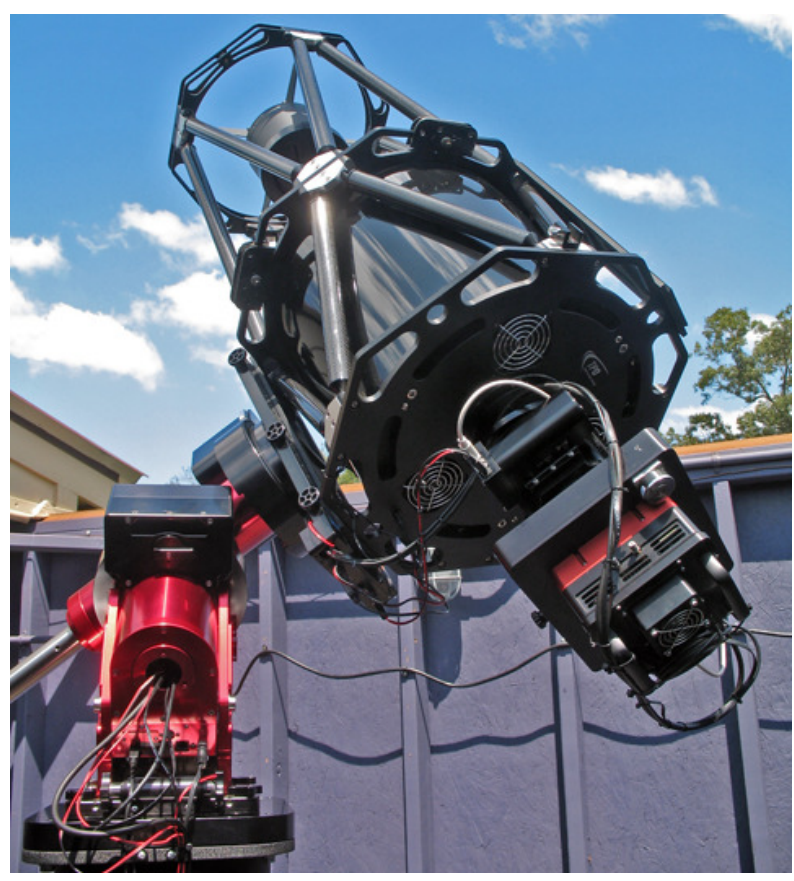

Fig. 3. Current telescope of Waffelow Creek Observatory: 0.356 RC telescope.

\section{BIRUNI OBSERVATORY}

This non- robotic observatory belongs to Shiraz university and located in longitude $\left(52^{\circ} 30^{\prime} 16^{\prime \prime} \mathrm{E}\right)$ and latitude $\left(29^{\circ} 39^{\prime} 01^{\prime \prime} \mathrm{N}\right)$ and $1740 \mathrm{~m}$ above sea level and $180 \mathrm{~m}$ from the general level of Shiraz city. The offer for the establishment of Biruni Observatory was proposed by Prof. Yousef Sobouti and Dr. Edward Francis Guinan in 1969. The operation of the construction of this observatory started in 1973 and it started operating in March 1977. This observatory reconstructed again in October 2017. For observation, this observatory has three domes. Two motorized spherical 3 meters domes and a 4.3 meters motorized spherical dome. The telescopes that these domes sheltering are two reflecting Schmidt Cassegrain telescopes 11 inches and inches and a 20 inches reflecting telescope of Cassegrain type which is the main telescope of this observatory (see Figures 4 and 5).

\subsection{Our Observation Instruments}

In this research the main instruments that we used are:

- 11 inches reflecting telescope of Schmidt Cassegrain type which is located under 3 meter motorized spherical dome.

- For imaging, we have used Meade DSI Pro II with resolution of $748 \times 577$. 


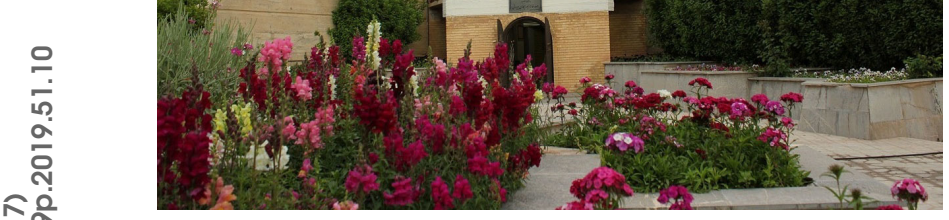

Fig. 4. Dome of 20 inches Cassegrain telescope.

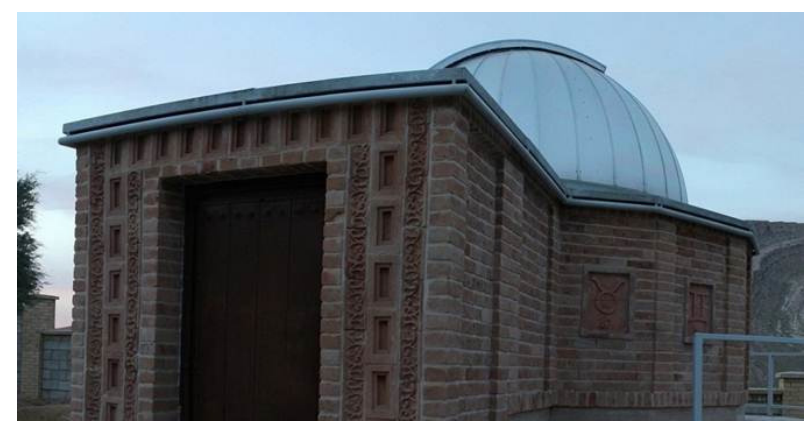

Fig. 5. Dome of 11 inches reflecting telescope of Schmidt Dome Cassegrain type.

\section{ECLIPSING VARIABLE STARS}

There are different factors that can cause variability of a star's light but in eclipsing variable star the reason of changing light is passing a component star in front of each other. We study star's properties by recording these changes on a curve which calls the light curve.

\subsection{Our Target Star}

Our target star in this study is BN Ari eclipsing variable star from Aries constellation. One can find this star with other names in different catalogs such as GSC 1761-1934, TYC 1761-1934-1, 2MASS J02090779+2629070 and AC2000 870736. Variability of this star discovered by Otero et al. in 2004 from data that released in the Northern Sky Variability Survey (NSVS). And in 2005 Pojmański et al. discovered BN Ari from data of All-Sky Automated Survey (ASAS) as a contact binary with the period of 0.299377 , the magnitude of 10.36 in the visual filter and the amplitude of variation of 0.73 .

For this study, our chosen comparison star is TYC 1761-2281-1 (GSC 1761-2281) with the right ascension of 02:09:28.81 and declination of $+26: 22: 29.960$.

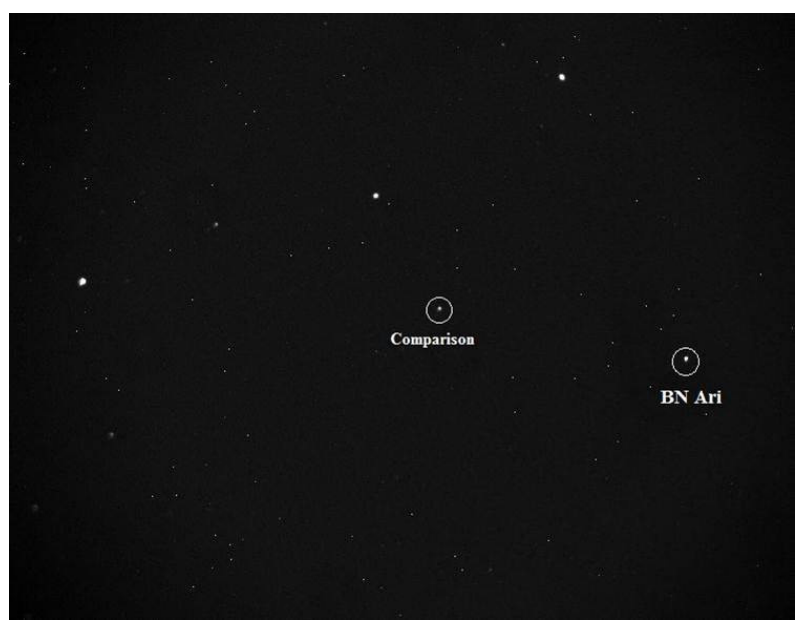

Fig. 6. Our target star, BN Ari, and its comparison star.

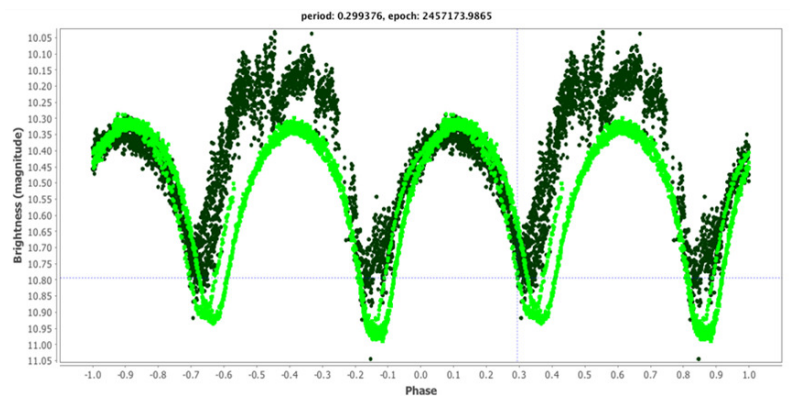

Fig. 7. Waffelow Creek robotic observatory and Biruni non-robotic observatory light curves of BN Ari eclipsing binary star.

In Figure 6 you can find BN Ari, its comparison and its surrounded stars in a field which we took with our observational instruments.

\section{COMPARING LIGHT CURVES}

By plotting Phase in Magnitude we found the light curve of BN Ari from our observations in Biruni none- robotic observatory in Red filter. Then, we asked Edward J. Michaels for some data from BN Ari with his robotic observatory and put both on a curve for comparing (Figure 7).

The dark green curve is Biruni observatory's light curve and the light green one is Waffelow Creek Observatory's light curve.

\section{DISCUSSION}

Michaels observation is in the visible filter and our observation is in the red filter that may be the reason of difference in place of light curves how that Biruni's light curve is a little above Waffelow Creek's 
light curve. Also, it's clear that dark green light curve seems chaotic mainly in picks and that can be because of air and light pollution in place of Biruni observatory. The light green light curve seems more arranged and that would be because of modernity of his devices. Anyway, this is not deniable the effect of changing the dome with hand and controlling the telescope and computer to align altogether had some effect on resulting line like light curves which Biruni observatory struggling with and Waffelow Creek observatory don't.

\section{REFERENCES}

Bordbar, G. H. \& Bahrani, F. 2016, RMxAC, 48, 118

Hasanzadeh, A., et al. 2015, Variable stars observation to analyze. Tehran, Iran: Daneshpazhoohane Javan publication

Michaels, E. J. 2015, JAAVSO, 43, 1

Ostli, D. A. \& Carroll, B. W. 1996, An Introduction to Modern Stellar Astrophysics, (Boston, MA: Pearson Addison Wesley)

Otero, S. A., Wils, P., \& Dubovsky, P. A. 2004, IBVS, 5570,1 